\title{
Daily ambulatory exhaled nitric oxide measurements in asthma
}

Pijnenburg MW, Floor SE, Hop WC, De Jongste JC. Daily ambulatory exhaled nitric oxide measurements in asthma.

Pediatr Allergy Immunol 2006: 17: 189-193.

(c) 2006 The Authors

Journal compilation @ 2006 Blackwell Munksgaard

Exhaled NO (FENO) is a non-invasive, validated marker for asthmatic airway inflammation. Recently, a new hand-held NO-analyzer has been developed which makes it possible to monitor FENO at home. We assessed feasibility and analyzed variability of daily FENO home measurements. Twenty-one asthmatics (mean age $14.5 \mathrm{yr}$; range 8 $25 \mathrm{yr}$ ) participated. Nineteen used a stable dose of inhaled corticosteroids and all of them were in a stable clinical condition. FENO was measured twice daily for 14 consecutive days. Measurements and symptom scores were recorded on a smart card in the analyzer. Symptom score items included well-being, wheeze, activity, and nocturnal symptoms. Measurements showed a success rate of $93 \%$. We found a significant diurnal variation in FENO with geometric mean morning levels $14 \%$ higher than evening levels (95\% CI: $4 \%-25 \%$; $\mathrm{p}=$ 0.013). Individual subjects showed marked fluctuation of FENO. The mean intrasubject coefficient of variation of FENO was $40 \%$ for morning and $36 \%$ for evening values. FENO and cumulative symptom scores did not correlate. Home FENO measurements are feasible, and offer the possibility to asses airway inflammation on a daily basis. Further study is needed to interpret and evaluate possible benefits of FENO home monitoring.

\author{
Mariëlle W. Pijnenburg', Suzanne E. \\ Floor ${ }^{1}$, Wim C. Hop ${ }^{2}$ and Johan C. De \\ Jongste ${ }^{1}$ \\ Departments of ${ }^{1}$ Pediatric Respiratory Medicine \\ ${ }^{2}$ Epidemiology and Biostatistics, Erasmus MC -Sophia \\ Children's Hospital, Erasmus University Medical \\ Centre, Rotterdam, The Netherlands
}

Key words: asthma; children; exhaled nitric oxide; home measurement; symptoms; variability

Mariëlle W Pijnenburg, Department of Pediatrics/ Pediatric Respiratory Medicine, Erasmus University Medical Centre - Sophia Children's Hospital, PO Box 2060, 3000 CB Rotterdam, The Netherlands Tel.: +31 104636263

Fax: +31104636772

E-mail: m.pijnenburg@erasmusmc.nl

Accepted 11 January 2006
The fraction of nitric oxide in exhaled air (FENO) correlates with eosinophils in induced sputum and with eosinophil infiltration of the airway wall in atopic asthmatics (1-5). This makes FENO a candidate marker to monitor asthmatic airway inflammation. In the clinic, FENO is routinely measured by chemiluminescence analyzers, which are expensive, not easily transportable, require frequent calibration and thus are not suitable for home monitoring. Recently, a new hand-held NO-analyzer (NIOX MINO $^{\text {TM }}$, Aerocrine, Solna, Sweden) was developed, which offers the possibility to measure FENO in the home situation. A good agreement between FENO tests with a conventional, approved NO-analyzer (NIOX, Aerocrine, Solna, Sweden) and NIOX MINO was observed in a recent study (6). Home monitoring of FENO has the potential to detect inflammation at an early stage and adapt treatment with inhaled steroids accordingly. This study was performed to evaluate feasibility and variability of FENO measurements with NIOX MINO ${ }^{\mathrm{TM}}$ at home. Second, we investigated the correlation between symptoms and FENO during the two weeks of the study.

\section{Methods}

Patients with doctor's diagnosed asthma, aged 6-25 yr were recruited during their outpatient clinic visit at the hospital. Patients should be in a stable clinical condition as judged by their own pediatric pulmonologist. Patients who had an exacerbation or used oral prednisone in the 3 months preceding the study, or who used rescue beta- 2 agonists more than four times a week in the $2 \mathrm{wk}$ preceding the study, patients who smoked and patients with a concurrent disease affecting FENO were excluded. Atopy 
was defined as RAST class 2 or higher for at least one inhalation allergen ever. The study was approved by the Medical Ethical Committee of the Erasmus University Medical Centre. In an open, observational, prospective study, patients measured FENO twice daily for $2 \mathrm{wk}$ at home. The NIOX MINO ${ }^{\mathrm{TM}}$ measures NO by an electrochemical sensor (Fig. 1). The measurement range is between 0 and $300 \mathrm{ppb}$ with an accuracy of $\pm 5 \mathrm{ppb}$ or $\pm 10 \%$ if FENO is $>30 \mathrm{ppb}$. Deep inhalation through the mouthpiece provides NO-free air via an NO-scrubber. Next, exhalation for $10 \mathrm{~s}$ with a constant flow of $50 \mathrm{ml} / \mathrm{s}$ is required. A visual and audible feedback system helps to maintain pressure within pre-set limits to ensure constant flow. Measurements are approved if exhaled pressure is between 10 and $20 \mathrm{~cm} \mathrm{H}_{2} \mathrm{O}$ with deviation allowed during the first $3 \mathrm{~s}$ of the measurement. For this study, there was no maximum for the number of allowed attempts. In previous studies NIOX MINO ${ }^{\mathrm{TM}}$ was validated and compared with the conventional NIOX NO-analyzer and showed good agreement $(6,7)$. NIOX MINO ${ }^{\mathrm{TM}}$ is provided with a smart card, which records all measurement results and time of measurements. The product we used also has a diary function where symptom scores can be recorded. Wellbeing, wheeze, activity, and nocturnal symptoms are scored on a 4-point scale (maximal daily score 12) and entered in an electronic diary. Data on FENO levels are available within minutes after the maneuver; symptom scores can be entered during these minutes. Patients were instructed by one researcher (SF) and were provided with a hand-held analyzer in case of satisfactory performance.

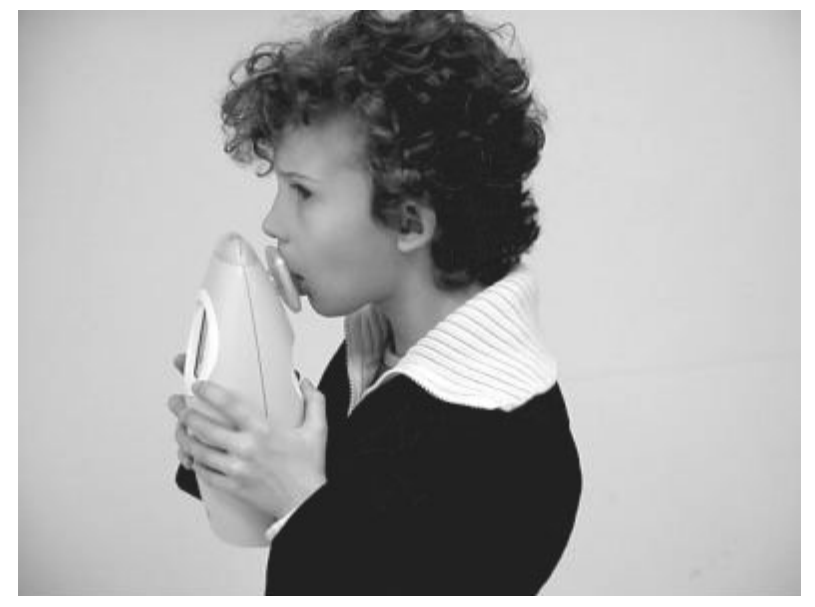

Fig. 1. Six-year-old patient performing a measurement of FENO with the NIOX MINO ${ }^{\mathrm{TM}}$.
Mixed Model ANOva was used to compare morning and evening FENO values and to investigate the correlation between FENO and cumulative symptom scores. In these analyses FENO values were log transformed. Spearman non-parametric correlation was used to assess the correlation of age with feasibility. Significance was assumed at $\mathrm{p}<0.05$. SPSS for Windows Version 10.1 and SAS (procedure PROC MIXED) were used for statistical analyses.

\section{Results}

Twenty-one asthmatics (11 males) were included. Their mean (range) age was $14.5 \mathrm{yr}$ (8.1-25.8), 18 of them were atopic. Lung function measurements showed a mean (range) FEV1 of $93 \%$ of predicted $(57 \%-120 \%)$ and a mean FVC of $100 \%(75 \%-124 \%)$. Nineteen used an inhaled steroid, median dose (range) $400 \mu \mathrm{g} /$ day budesonide or equivalent (200-1600). Of those, 12 used a long-acting beta agonist as well.

Of the maximum number of 588 FENO measurements (21 subjects, twice daily for 2 wk) 44 values were missing, which gives a success rate of $93 \%$. Non-adherence was the main cause of missing values. The success rate was age-dependent, in the way that older age was associated with worse adherence $(\mathrm{r}=-0.56, \mathrm{p}=$ 0.008). The only technical problem requiring intervention of the researchers was sensor failure in one analyzer.

Only three patients were non-atopic and as atopy might affect variability of FENO and the correlation with symptoms, these patients were excluded from further analyses. Two of them used a combination of inhaled steroids and a long-acting beta- 2 agonist, the other one did not use any medication. Geometric mean morning FENO in the remaining 18 patients was $14 \%$ higher compared to evening levels (95\% CI: 4\%$25 \% ; \mathrm{p}=0.013$ ) (Fig. 2). There was marked fluctuation of FENO in individual patients with a mean coefficient of variation $(\mathrm{CV})$ of $40 \%$ for morning values (range 18\%-93\%) and $36 \%$ for evening values (range 15-72). The difference in coefficient of variation for morning and evening values was not significant $(\mathrm{p}=0.35$, Wilcoxon signed ranks test).

Overall, no significant correlation between the mean daily symptom scores and geometric mean FENO-levels was found (Fig. 3). In the same way, within-patients between-days changes of FENO did not correlate with changes of cumulative symptoms on following days.

Analysis of the results within individuals showed a number of patterns, of which examples 


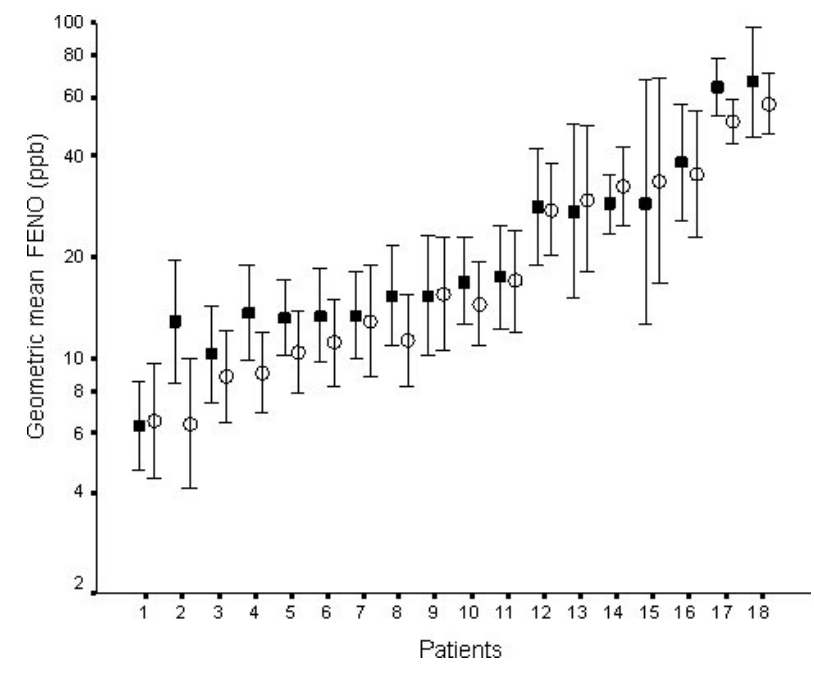

Fig. 2. Individual diurnal variability of geometric mean FENO for each patient. Morning values (closed symbols) and afternoon values (open symbols) \pm 1 s.d. for each patient during $2 \mathrm{wk}$. The mean diurnal variation of all patients was $14 \%(95 \%$ CI $4 \%-25 \%, \mathrm{p}=0.013)$.

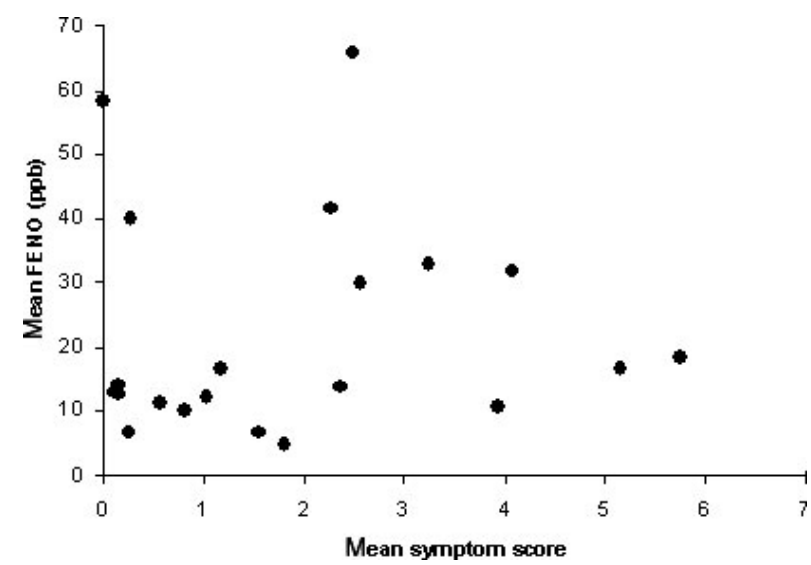

Fig. 3. Mean daily cumulative symptom scores (vertical axis) as a function of geometric mean FENO-levels. Each symbol represents a patient. The correlation was not significant.

are shown in Fig. 4. Most children showed stable, low FENO with either stable, low-symptom scores $(\mathrm{n}=8)$ (Fig. $4 \mathrm{a})$ or high, sometimes widely varying symptom scores $(n=7)$ (Fig. $4 b)$. Five showed evident parallel changes of FENO and symptoms over several days: one of them started measuring immediately upon returning from a school camp and showed a gradual normalization of extremely high FENO within $2 \mathrm{wk}$, paralleled by a reduction in symptoms (Fig. 4c). Another child visited a similar camp during the $2 \mathrm{wk}$ of monitoring; afterward both FENO and symptoms had risen dramatically (Fig. 4d). One patient showed high stable FENO with low symptoms (Fig. 4e), and one high stable FENO with high symptom scores (Fig. 4f).

\section{Discussion}

This is the first report on FENO measurements taken by asthmatic patients in their homes. Until now, the bulky and complicated NO chemiluminescence analyzers have limited the applicability of FENO as an instrument for frequent monitoring of airway inflammation in asthma. The new hand-held analyzer was successfully used for daily home measurements of FENO during $2 \mathrm{wk}$, without professional support. We found significant diurnal FENO variability with highest values in the morning, and considerable dayto-day variation. Mean FENO values and changes in FENO between days did not correlate with mean symptom scores within the time frame of the study; individual results were suggestive of parallel changes in some children as a result of external factors.

Others and we have shown that FENO correlates with eosinophilic airway inflammation, assessed in induced sputum or airway biopsies in atopic asthmatic adults and children (1-5). Treatment with corticosteroids reduces FENO levels in asthmatic patients in a dose-dependent manner $(8,9)$. Measuring FENO at home on a daily basis offers the possibility to detect changes in inflammation in an early stage and to adjust treatment accordingly.

We demonstrated a marked diurnal FENO variation. This is in line with findings by Mattes et al., who found a statistical trend towards higher morning FENO values and a cosine like circadian rhythm of FENO, for asthmatic as well as a small number of healthy children (10). Ten Hacken et al. found higher FENO at 4 a.m. compared to 4 p.m. in adult patients with nocturnal asthma and peak flow variability of $>15 \%$ (11). In contrast, Kharitonov et al. found no diurnal or day-to-day variation in healthy and asthmatic adults and children during a 5-day follow-up (12). The mechanism of diurnal FENO variation is still unclear. Both variation in the inflammatory process and external factors like sleep and food intake might play a role. For the moment, it seems important to perform serial FENO measurements within patients at the same time of the day.

In the present study, day-to-day variation of FENO was common, however, especially in the lower (normal) FENO ranges this variability is clinically not relevant. Although we tried to include patients with stable asthma, almost half of them experienced clinical instability during the 

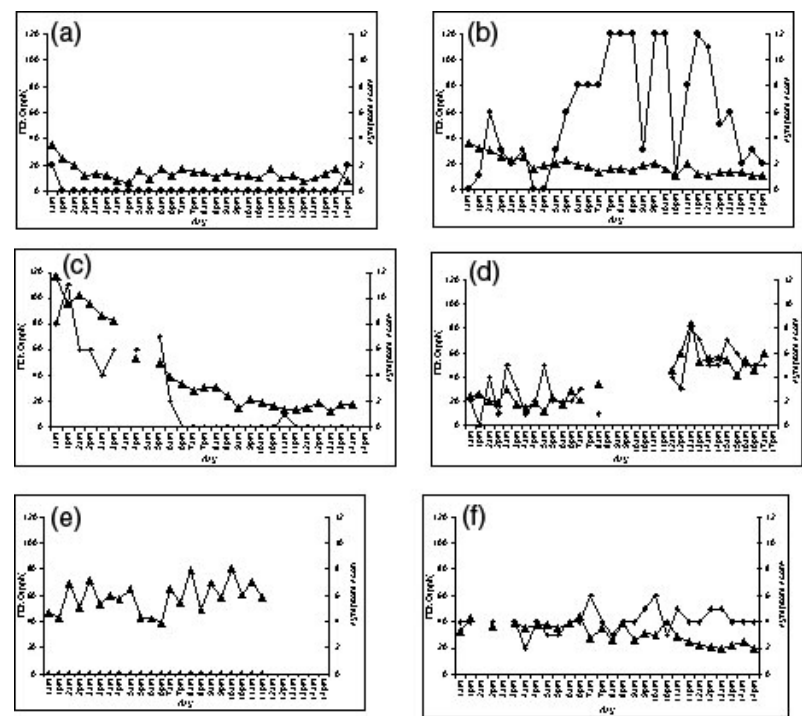

Fig. 4. Six examples of individual FENO and symptom scores. On the $x$-axis time in days, on the y-axis FENO (left) and cumulative symptom scores (right). (a) Low FENO with low symptom scores. (b) Low FENO with varying symptom scores. (c) Parallel change of FENO and symptoms: this child started measurements after returning from a camp. Both FENO and symptoms start high and normalize within 2 wk. (d) Parallel change of FENO and symptoms: a child who went to a school camp during the $2 \mathrm{wk}$ of measurement, missed some measurements for that reason and showed a marked increase in both symptoms and FENO upon return home. (e) High FENO with low symptoms. (f) High FENO with high symptom scores.

study and in some cases an external trigger seemed responsible for parallel changes in FENO and symptoms (Fig. 4). This clinical instability may at least partly explain the high day-to-day variation we found in this study. On the contrary, some individuals showed marked instability of symptoms with stable, low FENO.

There is a good agreement between FENO measurements with NIOX MINO and a conventional chemiluminescence analyzer. In this study, an average disagreement of $0.5 \mathrm{ppb}$ with a mean standard deviation of $3.8 \mathrm{ppb}$ was found from 251 measurements in 19 subjects (6). This makes it very unlikely that the diurnal variability may be explained by variability of the analyzer itself.

Our study was not designed to answer the question if home monitoring of FENO might predict clinical instability. Earlier studies on the correlation of FENO and asthma severity and control show conflicting results. These may be due to different FENO measuring techniques, patient selection criteria and the use of ICS (13-20). However, some recent studies including our own showed that FENO might be used as a 'loss of control' marker, predicting asthma exacerbations or relapse after cessation or reduction of ICS (21-23). In the study of Jones, single measurements and changes of FENO from baseline had positive predictive values of $80 \%-$ $90 \%$ for predicting and diagnosing loss of asthma control (22). In a previous study, we followed children with clinical remission of asthma who were taken off ICS; FENO at 2 and $4 \mathrm{wk}$ after withdrawal predicted asthma relapse in the forthcoming months (23). We speculate that the lack of a correlation between symptoms and FENO in the present study may be due to its limited time frame. The question is whether FENO might be used to adapt ICS doses, and whether or not frequent home monitoring has advantages above infrequent assessment at clinic visits. The relevance of FENO fluctuations for asthma management is clearly important to elucidate.

We conclude that ambulatory FENO measurements with NIOX MINO ${ }^{\mathrm{TM}}$ are feasible, and offer the possibility to measure asthmatic inflammation frequently at home. Morning values are significantly higher than evening values and considerable day-to-day variability exists, partly due to clinical instability. Longer follow-up studies are now warranted to evaluate whether and how frequent FENO measurements may be used to improve asthma management.

\section{Acknowledgments}

The authors are grateful to the Netherlands Asthma Fund and the Van Stolk Fonds for their financial support, and to Aerocrine for providing the NO analyzers.

\section{References}

1. Piacentini GL, Bodini A, Costella S, et al. Exhaled nitric oxide and sputum eosinophil markers of inflammation in asthmatic children. Eur Respir J 1999: 13: 1386-90.

2. Van den Toorn LM, Prins JB, Overbeek SE, HooGSTEDEN HC, De Jongste JC. Adolescents in clinical remission of atopic asthma have elevated exhaled nitric oxide levels and bronchial hyperresponsiveness. Am J Respir Crit Care Med 2000: 162: 953-57.

3. Berlyne GS, Parameswaran K, Kamada D, Efthimiadis A, Hargreave FE. A comparison of exhaled nitric oxide and induced sputum as markers of airway inflammation. J Allergy Clin Immunol 2000: 106: 638-44.

4. Payne DN, Adcock IM, Wilson NM, OAtes T, SCALlan M, Bush A. Relationship between exhaled nitric oxide and mucosal eosinophilic inflammation in children with difficult asthma, after treatment with oral prednisolone. Am J Respir Crit Care Med 2001: 164: 1376-81.

5. VAn den Toorn LM, Overbeek S, De Jongste JC, Leman K, Hoogsteden HC, Prins JB. Airway inflammation is present during clinical remission of atopic asthma. Am J Respir Crit Care Med 2001: 164: 2107113. 
6. Hemmingsson T, Linnarsson D, Gambert R. Novel hand-held device for exhaled nitric oxide analysis in research and clinical applications. J Clin Monit 2004: 18: 379-87.

7. Alving K, Nordvall Sl, Janson C, Pedroletti C. Agreement between a stationary device (NIOX) and a new hand-held device (NIOX MINO) for fractional exhaled nitric oxide (FeNO) measurements in adults and children. Eur Respir J 2004: 24: 163S.

8. Tsai YG, Lee MY, Yang KD, Chu DM, Yuh YS, Hung CH. A single dose of nebulized budesonide decreases exhaled nitric oxide in children with acute asthma. J Pediatr 2001: 139: 433-37.

9. Kharitonov SA, Yates DH, Barnes PJ. Inhaled glucocorticoids decrease nitric oxide in exhaled air of asthmatic patients. Am J Respir Crit Care Med 1996: 153: 454-57.

10. Mattes J, Storm van's Gravesande K, Moeller C, Moseler M, Brandis M, KueHr J. Circadian variation of exhaled nitric oxide and urinary eosinophil protein $\mathrm{X}$ in asthmatic and healthy children. Pediatr Res 2002: 51: 190-94.

11. TEN HACKen NH, van der VAart H, van der Mark TW, Koeter GH, Postma DS. Exhaled nitric oxide is higher both at day and night in subjects with nocturnal asthma. Am J Respir Crit Care Med 1998: 158: 902-7.

12. Kharitonov SA, Gonio F, Kelly C, Meah S, Barnes PJ. Reproducibility of exhaled nitric oxide measurements in healthy and asthmatic adults and children. Eur Respir J 2003: 21: 433-38.

13. Artlich A, Busch T, Lewandowski K, Jonas S, Gortner L, FAlKe KJ. Childhood asthma: exhaled nitric oxide in relation to clinical symptoms. Eur Respir J 1999: 13: 1396-401.

14. Sippel JM, Holden WE, Tilles SA, et al. Exhaled nitric oxide levels correlate with measures of disease control in asthma. J Allergy Clin Immunol 2000: 106: 645-50.

15. Roberts G, Hurley C, Bush A, Lack G. Longitudinal study of grass pollen exposure, symptoms, and exhaled nitric oxide in childhood seasonal allergic asthma. Thorax 2004: 59: 752-56.

16. PAYNe DN, QIU Y, Zhu J, et al. Airway inflammation in children with difficult asthma: relationships with airflow limitation and persistent symptoms. Thorax 2004: 59: 862-69.

17. Meyts I, Proesmans M, De Boeck K. Exhaled nitric oxide corresponds with office evaluation of asthma control. Pediatr Pulmonol 2003: 36: 283-89.

18. Olin AC, Alving K, Toren K. Exhaled nitric oxide: relation to sensitization and respiratory symptoms. Clin Exp Allergy 2004: 34: 221-26.

19. Delgado-Corcoran C, Kissoon N, Murphy SP, DUCKWORTH LJ. Exhaled nitric oxide reflects asthma severity and asthma control. Pediatr Crit Care Med 2004: 5: 48-52.

20. Warke TJ, Mairs V, Fitch PS, McGovern V, Ennis M, SHIElds MD. Exhaled nitric oxide in relation to the clinical features of childhood asthma. J Asthma 2004: 41: 745-51.

21. Jatakanon A, Lim S, Barnes PJ. Changes in sputum eosinophils predict loss of asthma control. Am J Respir Crit Care Med 2000: 161: 64-72.

22. Jones SL, Kittelson J, Cowan JO, et al. The predictive value of exhaled nitric oxide measurements in assessing changes in asthma control. Am J Respir Crit Care Med 2001: 164: 738-43.

23. Pijnenburg MW, Hofhuis W, Hop WC, De Jongste JC. Exhaled nitric oxide predicts asthma relapse in children with clinical asthma remission. Thorax 2005: 60: 215-18. 